\title{
Arterial Trip Length \\ Characteristics Software
}
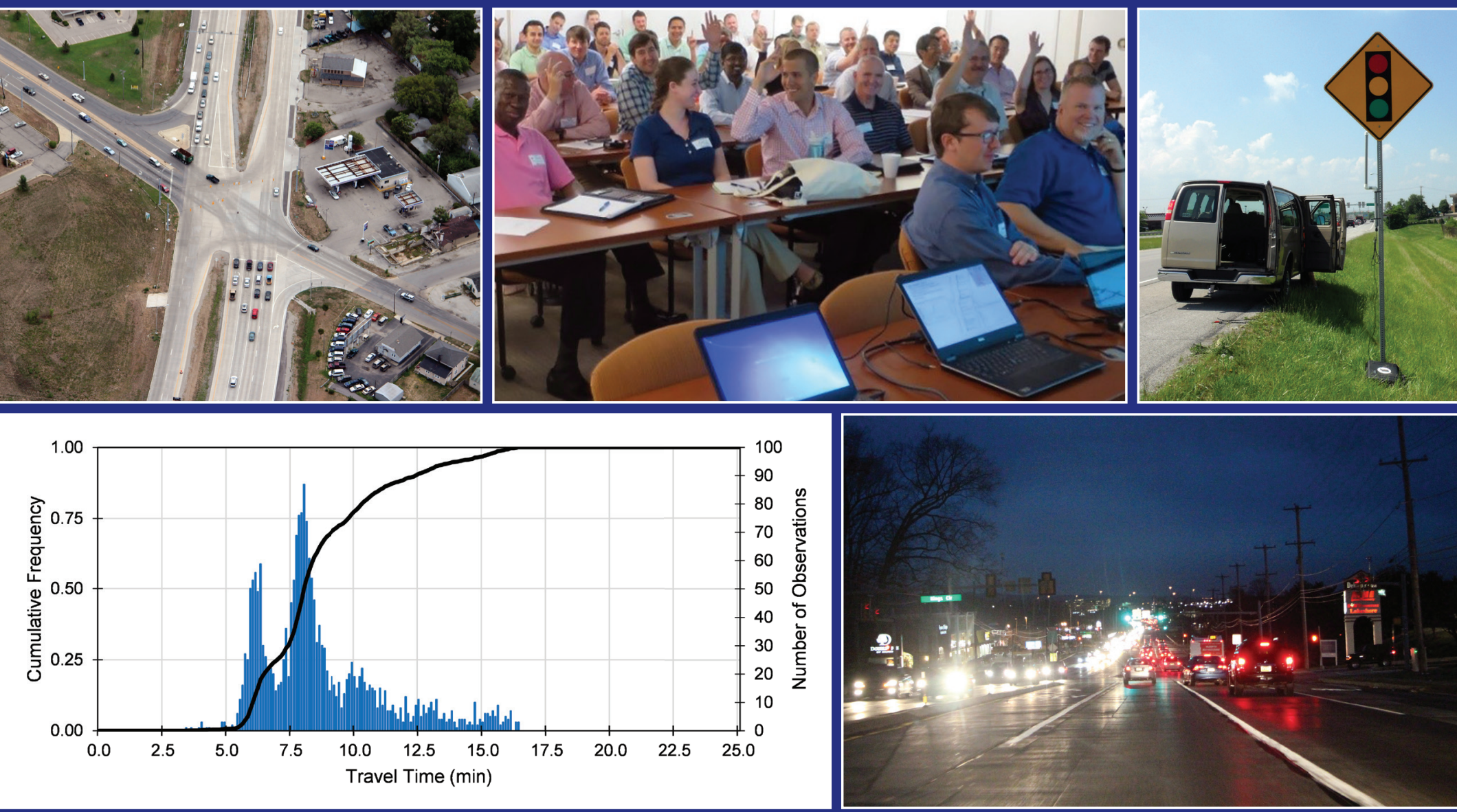

Stanley E. Young, Dennis So Ting Fong 


\title{
Arterial Trip Length Characteristics Software
}

\author{
Stanley E. Young \\ Traffax, Inc. \\ Dennis So Ting Fong \\ Traffax, Inc.
}

SBIR Phase 3 Joint Transportation Research Project

Traffax, Inc.

Purdue University

July 3, 2017

\begin{tabular}{|l|l|}
\hline Deliverable Reference: & D2.5 Accessibility Metrics Report \\
\hline Project Name: & $\begin{array}{l}\text { Sensor Fusion and MOE Development for Off-Line } \\
\text { Traffic Analysis of Real Time Data }\end{array}$ \\
\hline Contractor: & Traffax, Inc. \\
\hline Contract Number: & DTFH61-14-C-00035 \\
\hline Contract Term Start & $9 / 4 / 2014$ \\
\hline Contract Term End & $9 / 4 / 2017$ \\
\hline Key Personnel & Stan Young, Darcy Bullock, Dennis So Ting Fong \\
\hline
\end{tabular}




\section{Recommended Citation}

Young, S. E., and D. S. T. Fong. Arterial Trip Length Characteristics Software. Purdue University, West Lafayette, Indiana, 2017. https://doi.org/10.5703/1288284316569

\section{Acknowledgments}

This work was supported by Traffax/USDOT SBIR DTFH6114C00035. The contents of this paper reflect the views of the authors, who are responsible for the facts and the accuracy of the data presented herein, and do not necessarily reflect the official views or policies of the sponsoring organizations. These contents do not constitute a standard, specification, or regulation. 


\section{Table of Contents}

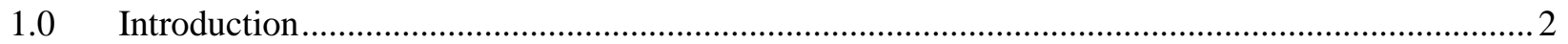

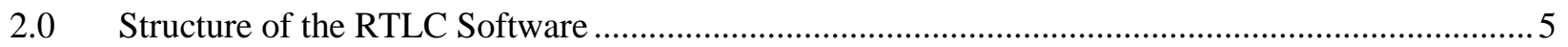

3.0 Software Implementation - Trip Chain Analysis ................................................................... 7

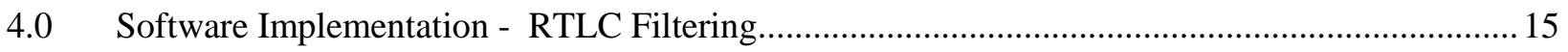

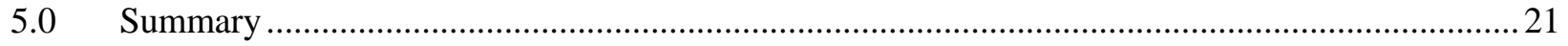

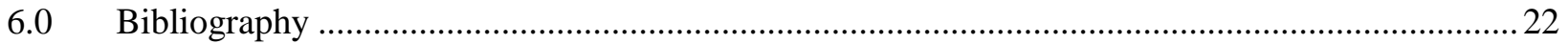

\section{Table of Figures}

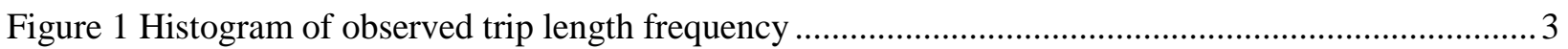

Figure 2 Cumulative frequency diagram of trip lengths contrasting two distinct corridors ........................ 4

Figure 3 Implementation of RTLC within BluSTATs.......................................................................... 5

Figure 4 Map of the Northern Virginia area showing locations of sensors …........................................... 7

Figure 5 Trip Analysis utility within BluSTATs which encompasses the RTLC metric ...........................9

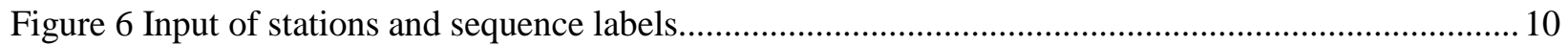

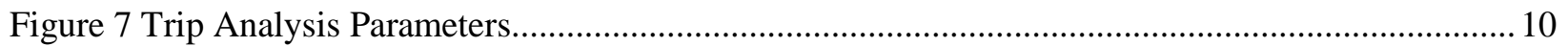

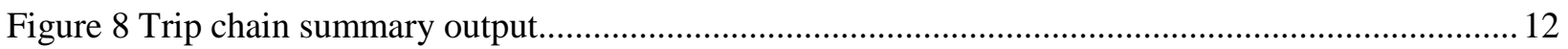

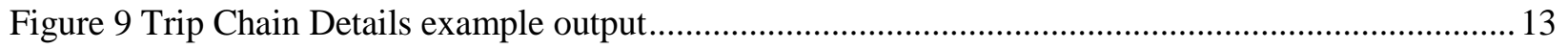

Figure 10 Additional filtering options for the RTLC analysis ................................................................ 15

Figure 11 Trip Analysis and RTLC options for an RTLC analysis for US1 case study ...........................16

Figure 12 Trip chain summary table for US-1 with RTLC filter options applied ................................... 17

Figure 13 Origin-Destination table of trips for the US-1 corridor ......................................................... 18

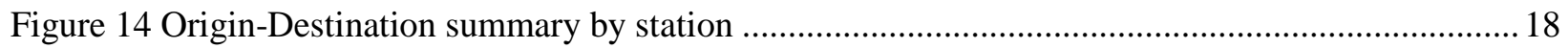

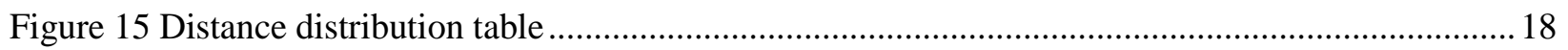

Figure 16 Histogram view of the RTLC trip distance distribution for US-1 .......................................... 19

Figure 17 Cumulative Frequency Diagram (CFD) view of the RTLC .................................................. 20

\section{List of Tables}

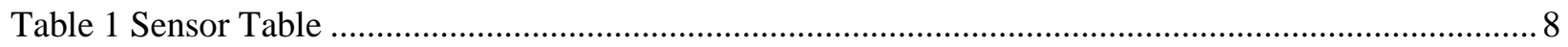

Table 2 Trip Chain Processing Logic …................................................................................................... 14 


\subsection{Introduction}

This report provides details of the software implementation for generation of roadway trip length characteristics (RTLC performance measure as originally set forth in September 2016 in a report entitled, “Arterial Trip Length Characteristics” [1]). The RTLC is an objective measure directly reflecting the subjective concept of throughput versus land access. It can supplant subjective judgment or indirect measures to provide a repeatable and defendable method for classifying roadways in order to compare and contrast operations, and proposed improvements and remediation. The RTLC can identify similarly functioning corridors across broad geographic spaces to be compared for various traffic, energy and safety performance measures. With modern re-identification equipment, the base data for RTLC can be collected cost effectively. The distribution of trip length can provide insight on roadway function at various times of day, which in turn can be compared with regulatory and/or signal timing to ensure they are in harmony.

In addition to the research that defined the RTLC, it is the objective of the project to provide reference implementations of the RTLC metric to enable consistent implementation. This report provides details of the algorithms, process, filters and programming methods for use in either procurement specification or development initiatives, and is designed as a reference document. The RTLC performance measure, introduced in the earlier report [1], has been programmed and included in the Traffax Inc. engineering software, BluSTATs. In previous software implementation, such as for arterial and network performance measures, open source reference code was provided that detailed the analytics and visualizations within the VPXplore open source analytics package. In the case of the RTLC such an approach was not feasible. Previous measures operated on travel time information reported at the segment level, and thus the CWS5200 data standard integrated into VPXplore was applicable. In contrast, the RTLC statistic is not developed from segment level travel time data. Rather it requires access to base sensor data collected at station prior to aggregation to segment level travel times. [Note that the implementation of the RTLC exposed the need for another layer of data standardization to port detection data at stations in a standard format. However, a station data standard is beyond the scope of the current project, and is identified for needed follow on research and development.]

The RTLC was implemented in BluSTATs where access to the sensor data at the station by station level is readily available. The analytics processing sequence for the production of the RTLC within BluSTATs are described in sufficient detail such that other programmers and analysts could develop like routines. This report reviews the implementation of RTLC within BluSTATs and provides an example of its execution in a reference case study.

\section{Review of the Roadway Trip Length Characteristic}

Methods to classify where a roadway falls on the mobility versus land access spectrum have traditionally relied on professional judgment based on geometric attributes. The Roadway Trip Length Characteristic (RTLC) provides a method to directly assess how the roadway is used by drivers. With modern reidentification equipment, the base data can be collected cost effectively. The distribution of trip length, as shown in Figure 1, can provide insight on roadway function at various times of day, which in turn can be compared with regulatory and/or signal timing to ensure they are in harmony. 


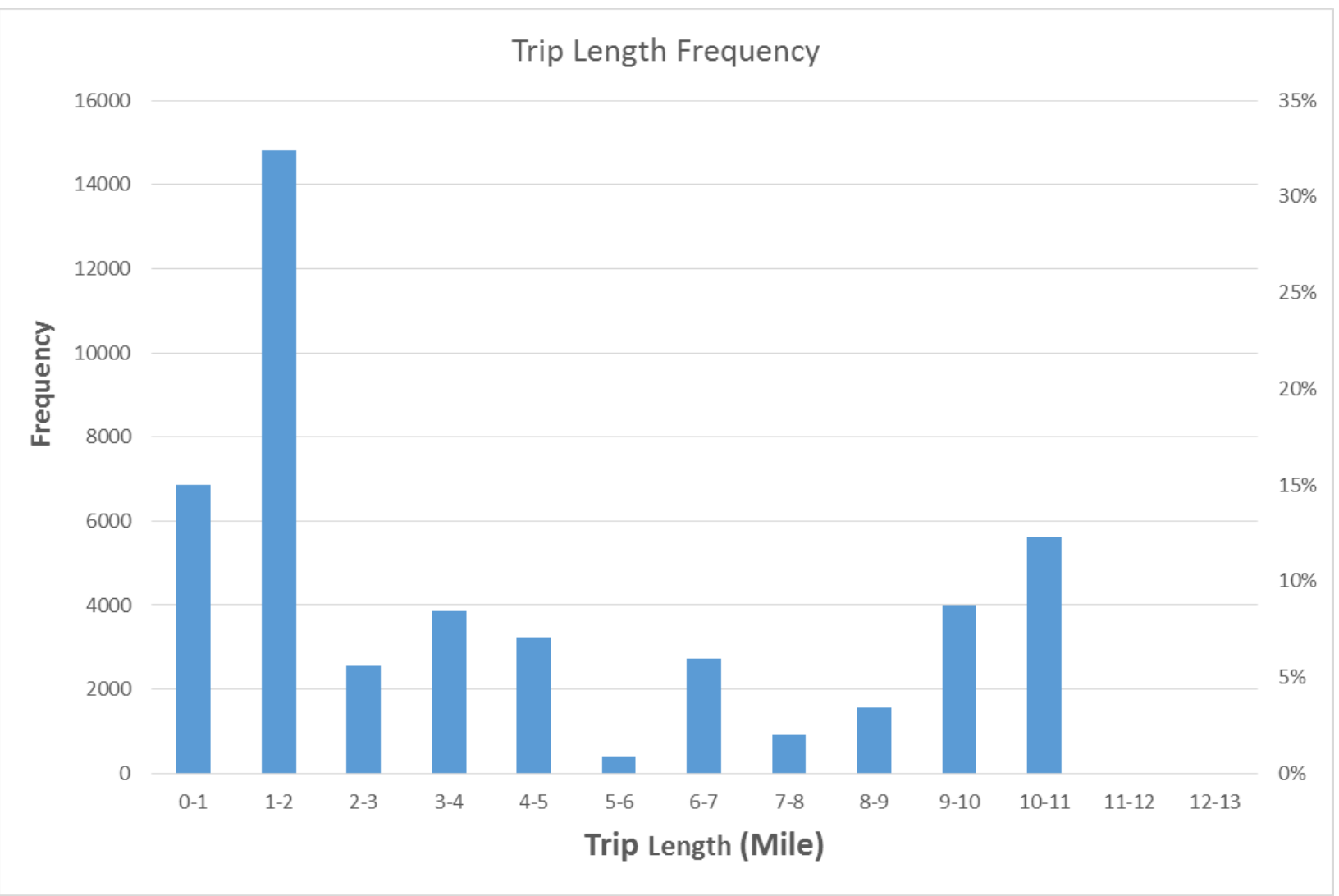

Figure 1 Histogram of observed trip length frequency

The trip length statistic is an objective measure directly reflecting the subjective concept of throughput versus land access. As a directly measurable attribute, it is useful for engineering and performance assessment processes. It can supplant subjective judgment or indirect measures to provide a repeatable and defendable method for classifying roadways in order to compare and contrast roadway operations. Moreover, it can also be used to find like roadways across broad geographical spaces to allow performance comparison as illustrated in Figure 2 below. This diagram contrasts RTLC between MD140 and NJ37; the two case studies presented in the earlier report [1]. 


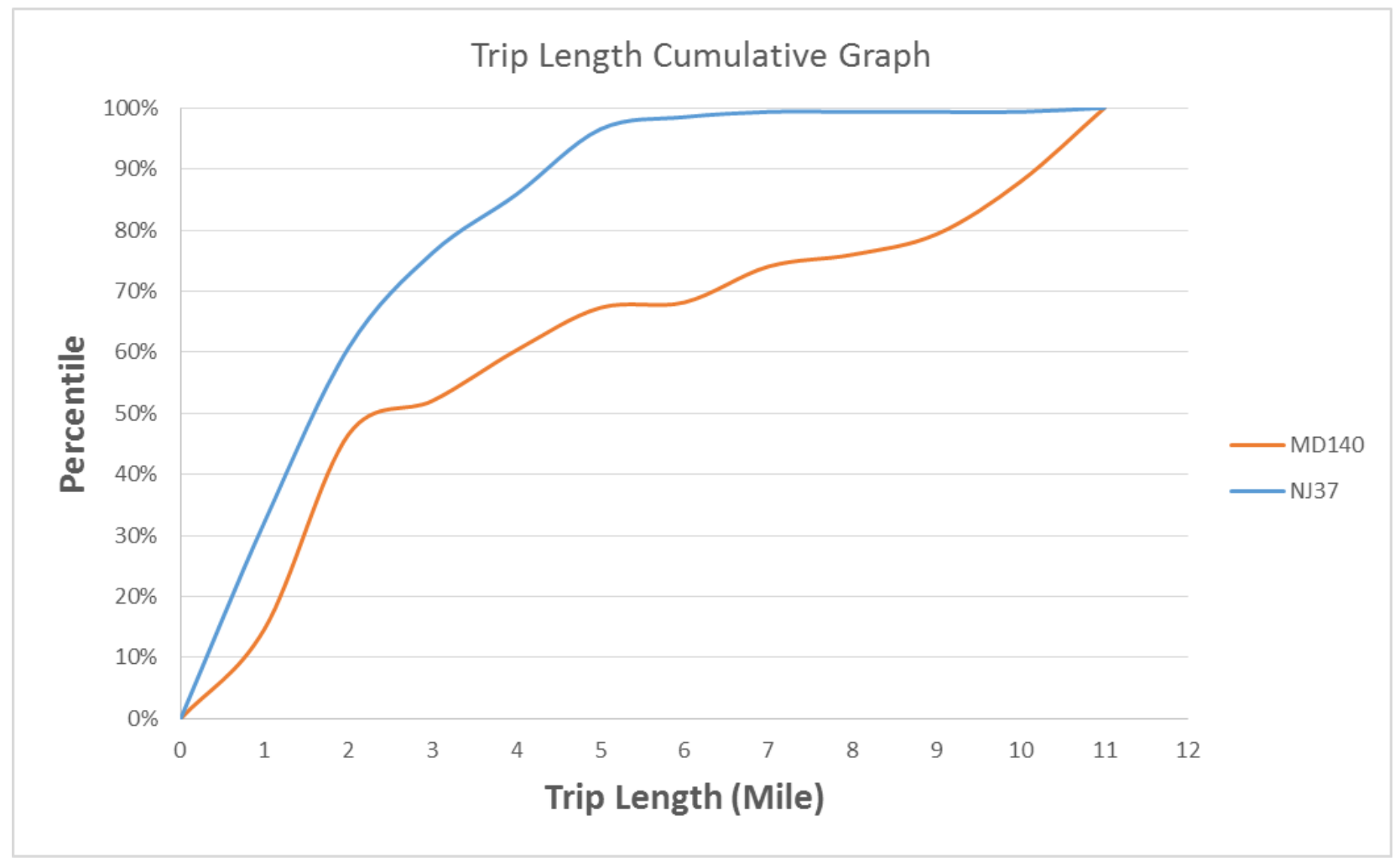

Figure 2 Cumulative frequency diagram of trip lengths contrasting two distinct corridors

In Figure 2, the cumulative frequency diagram (CFD) of trip length indicates that through movements are a dominant use of Maryland Route 140, whereas the trip lengths from NJ37 were more evenly distributed across multiple trip lengths. Data and inferences gained from the RTLC method allow for comparisons of vehicle traffic operations either on the same facility (different times of day) or between two different facilities. These comparisons provide a valuable tool for management policy, signal timing and coordination schemes, and other operations treatments.

Note, although the RTLC was constructed and demonstrated using Bluetooth re-identification data, it is not restricted to any particular type of re-identification data, nor is it strictly applicable only to reidentification data. As modern data sources continue to mature, individual vehicle location data (also called traces) can also be used as the basis for calculating RTLC information. Data sources such as vehicle probe based traffic data and vehicle-to-vehicle dedicated short-range communication are anticipated to provide such data in the future. As such, the methods provided herein can be generalized and adapted to new data sources.

The RTLC method provides a repeatable and objective procedure to characterize roadway function based on how it is used by drivers. The RTLC also complements classification by design attributes and allows for a higher granularity approach to roadway characterization. 


\subsection{Structure of the RTLC Software}

The implementation of the RTLC is carried out in the BluSTATs software, owned and maintained by Traffax Inc., and distributed in a compiled form for the Windows ${ }^{\mathrm{TM}}$ operating environment. The RTLC operates on detection data from the sensors within a corridor before it is aggregated to segment level travel times. As no standard data format exists for porting re-identification sensor data for stations, the RTLC software was implemented directly in BluSTATs where it can directly access the Traffax station data structures.

The analytics processing is detailed in this report for others to easily implement. The goal is to provide a reference for implementation, sort of a standard recipe, for the RTLC. A diagram of the approach is shown in Figure 3 below.

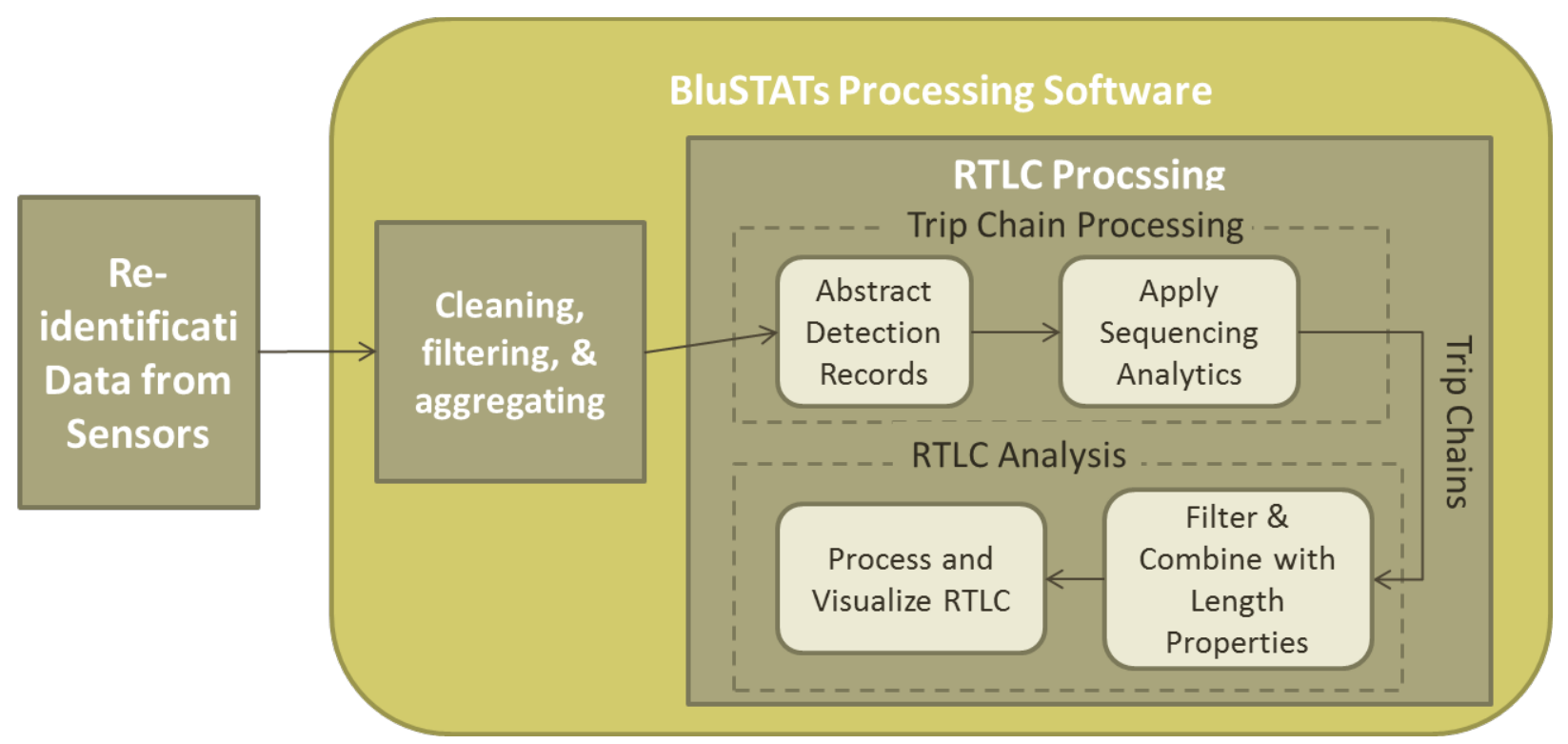

Figure 3 Implementation of RTLC within BluSTATs

Figure 3 illustrates the sequence of analytics processing within BluSTATs. Data from field sensors are input into BluSTATs. Station processing performs cleaning, filtering and aggregating to provide detection data in a station data structure format. Station detection data sets are then input into the RTLC processing which consists of two processing steps as explained below.

Processing Steps

Trip Chain Processing The stations are first labeled according to their sequence along a corridor. Although station records indicate the position of the sensor in spatial coordinates (latitude and longitude), it contains no information of the order of the sensors along a route. The first step in RTLC processing is sequencing the stations and providing sequential labels (A,B,C,D,E ...etc). These labels provide a short hand notation which enables efficient trip chain demarcation expressed simply,as ABC or DCBA, etc. Station trip chains are then analyzed using sequencing analytics. The end result is an exhaustive list of each trip along the corridor expressed in a way that shows the order and time when the vehicle was detected at each station. 
For example, a trip chain of DEFGH indicates that a vehicle was first detected at sensor station $D$, then $E$, then $F$, then $G$, and lastly $H$.

RTLC Analysis The RTLC analysis is performed on the exhaustive list of trip chains. First filtering is applied to limit the trips to those only of interest in the corridor (time of day, day of week, and other sequencing filters to be explained later) A distance metric is calculated for each trip and recorded with the trip chain. Frequency of trips is then combined with the trip length to create the RTLC measure. The results of the RTLC are then presented both in tabular format as well as statistical visualizations such as histograms and cumulative frequency diagrams (CFDs) of trip length.

The end result of the RTLC software is standard data representations in the form of tables and matrices, from which various RTLC graphics may be easily devised. The software in BluSTATs provides both histogram and CFD representation. The data can also be output to standard spreadsheet tools for the user to produce custom graphics as needed.

The software implementation is divided into two parts consistent with the processing flow in Figure 3. The RTLC is built upon a trip analysis utility within BluSTATs which creates trip chains. The trip analysis tool and its functionality are described in chapter 3 . Once a set of station data records have been analyzed for trip chains, the RTLC is analyzed for a corridor. Note that trip chains can be assessed for any configuration of sensor stations (corridor, network, etc.). The RTLC is specific to a corridor, requiring distance information between sensors, and some additional filtering of trip chains. The processing steps applied to the trip chain records to create the RTLC statistics and visualization, are described in detail in chapter 4.0. 


\subsection{Software Implementation - Trip Chain Analysis}

The detailed implementation of the software is illustrated through use of a case study. The case study comes from US Route 1 in Northern Virginia. A map of the area and the sensor locations are given in Figure 4. This data was collected during the last three weeks of October of 2011.

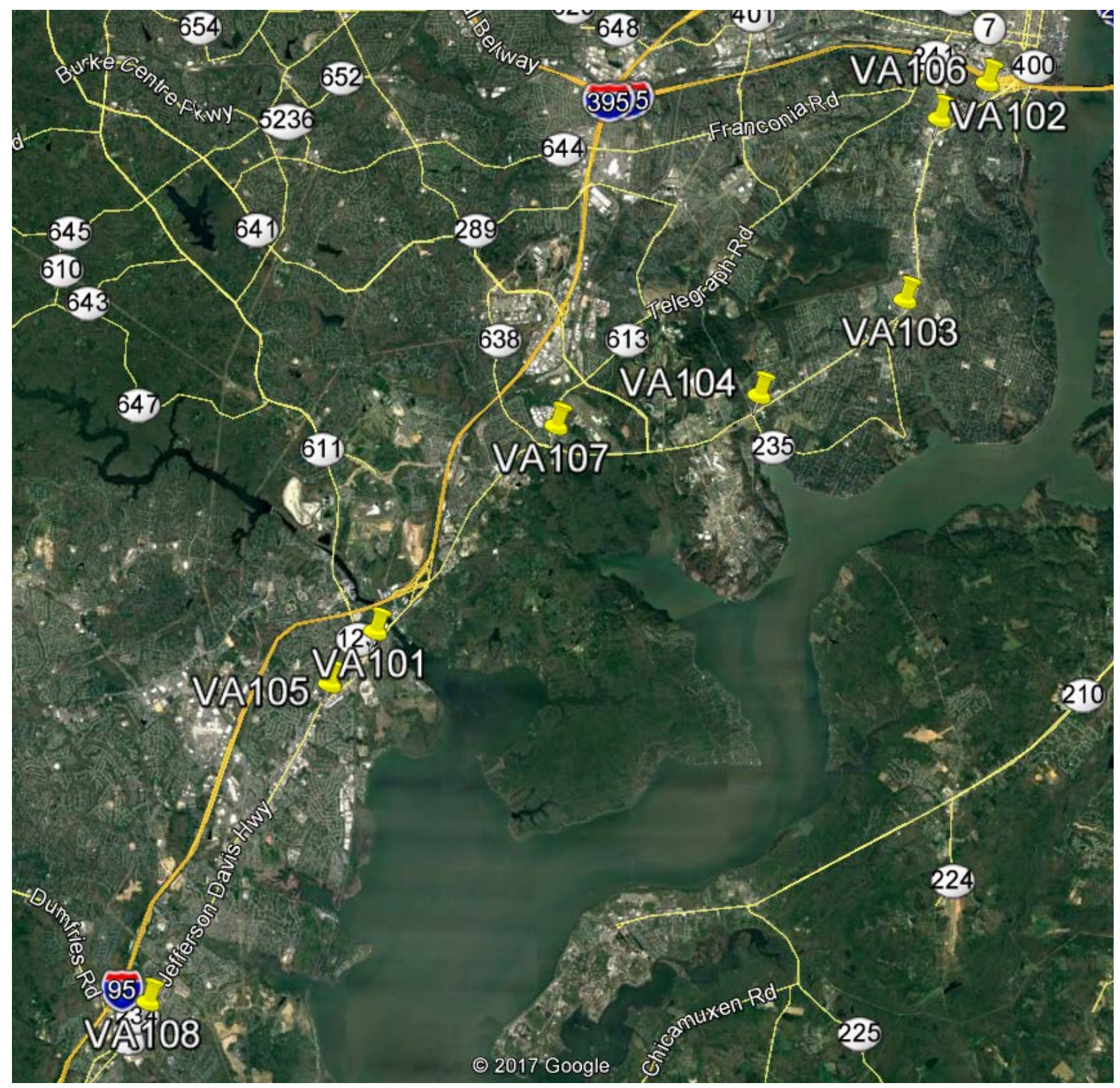

Figure 4 Map of the Northern Virginia area showing locations of sensors 
Note the sequence of sensors, from the upper right to the lower left in Figure 4, is as follows:

Table 1 Sensor Table

\begin{tabular}{|l|c|c|c|}
\hline Sensor Label & Sequence Label & $\begin{array}{c}\text { Distance to Previous } \\
\text { Station (miles) }\end{array}$ & $\begin{array}{c}\text { Cumulative Distance } \\
\text { (miles) }\end{array}$ \\
\hline VA106 & A & 0 & 0 \\
\hline VA102 & B & 1.0 & 1.0 \\
\hline VA103 & C & 3.06 & 4.06 \\
\hline VA104 & D & 2.82 & 6.88 \\
\hline VA107 & E & 3.45 & 10.33 \\
\hline VA101 & F & 4.58 & 14.91 \\
\hline VA105 & G & 1.15 & 16.06 \\
\hline VA108 & H & 5.96 & 22.02 \\
\hline
\end{tabular}

The sensors (referred to as stations within the BluSTATs software and hereafter) are listed in the correct sequence order in the first column progressing from the furthest north to the furthest south along US Route 1. The sequence label is a single letter (can also be a single digit number or other alpha-numeric character). In this case study, station VA106 is assigned the sequence label 'A', sensor VA102 is assigned the sequence label ' $B$ ' and so on through sensor VA108 which is labeled ' $H$ '. Note that sensor VA101 follows sensor VA107 and comes before sensor VA105 in the sequence order along the route, although the labels of the sensors seem to indicate some other order. For the RTLC system to work properly, the software needs to understand the sequencing of the stations as listed in the table, as well as the distance between sensors.

The BluSTATs software prompts for this information in the input screens. The RTLC software utility is located under Station Data Utilities, selectable from the Stations Processing screen. Note that the input and processing of field data in BluSTATs is covered in the BluSTATs Manual, available from Traffax Inc. Only the steps for creating the RTLC from processed station data are included in this report. This includes both the creation and analysis of trip chains combined with filtering and distance information to create RTLC metrics. From the Station Data Utilities screen, select Trip Analysis. Once selected, the Trip Analysis screen will appear as shown in Figure 5. This is the screen for input of the parameters for all aspects of the RLTC analysis. 


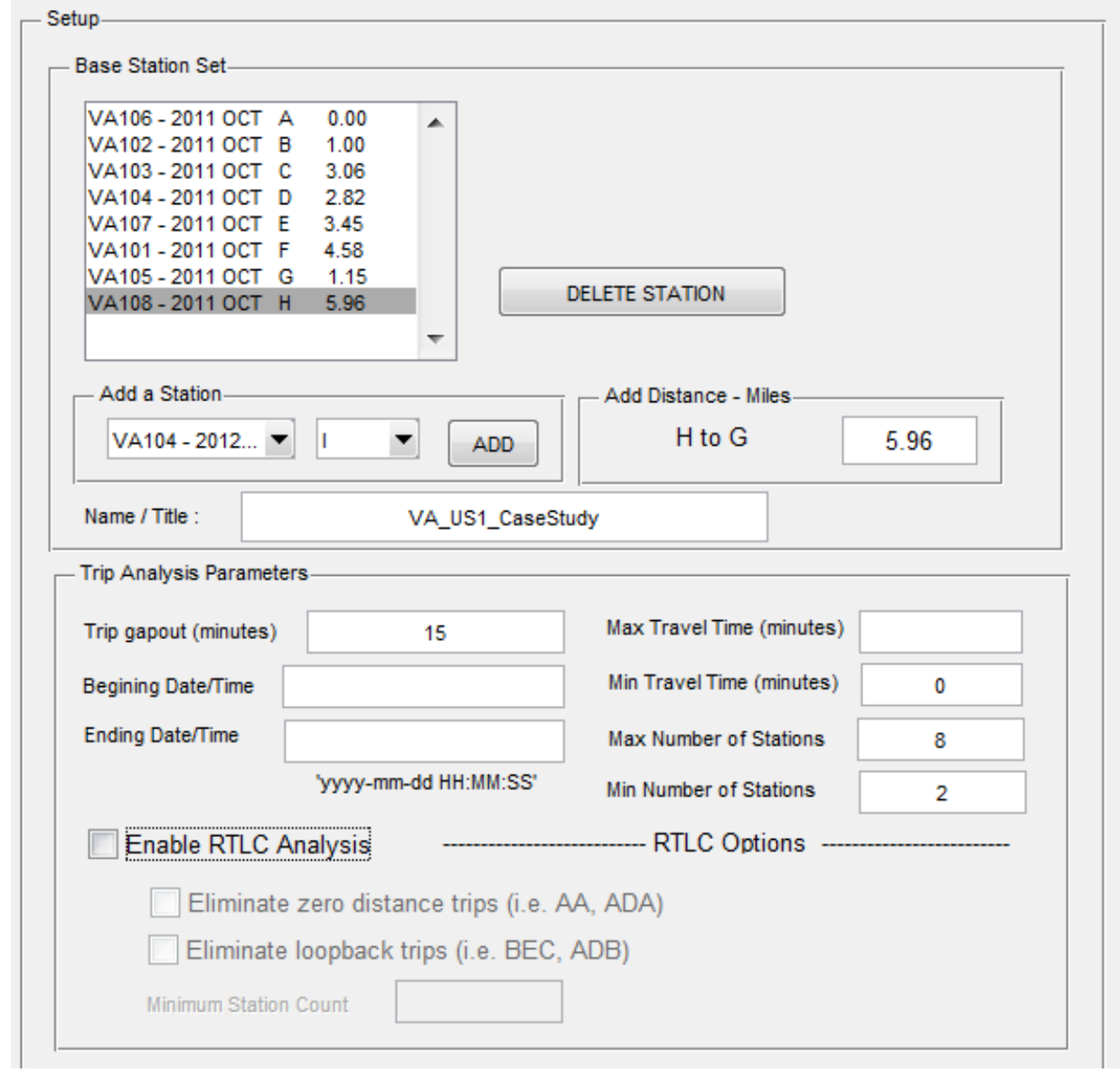

Figure 5 Trip Analysis utility within BluSTATs which encompasses the RTLC metric

The options in the upper left of the Trip Analysis screen identify stations and assign their sequence label. The inset in Figure 6 illustrates the selection of VA106 - 2011 OCT as position 'A' in the sequence for the analysis. 


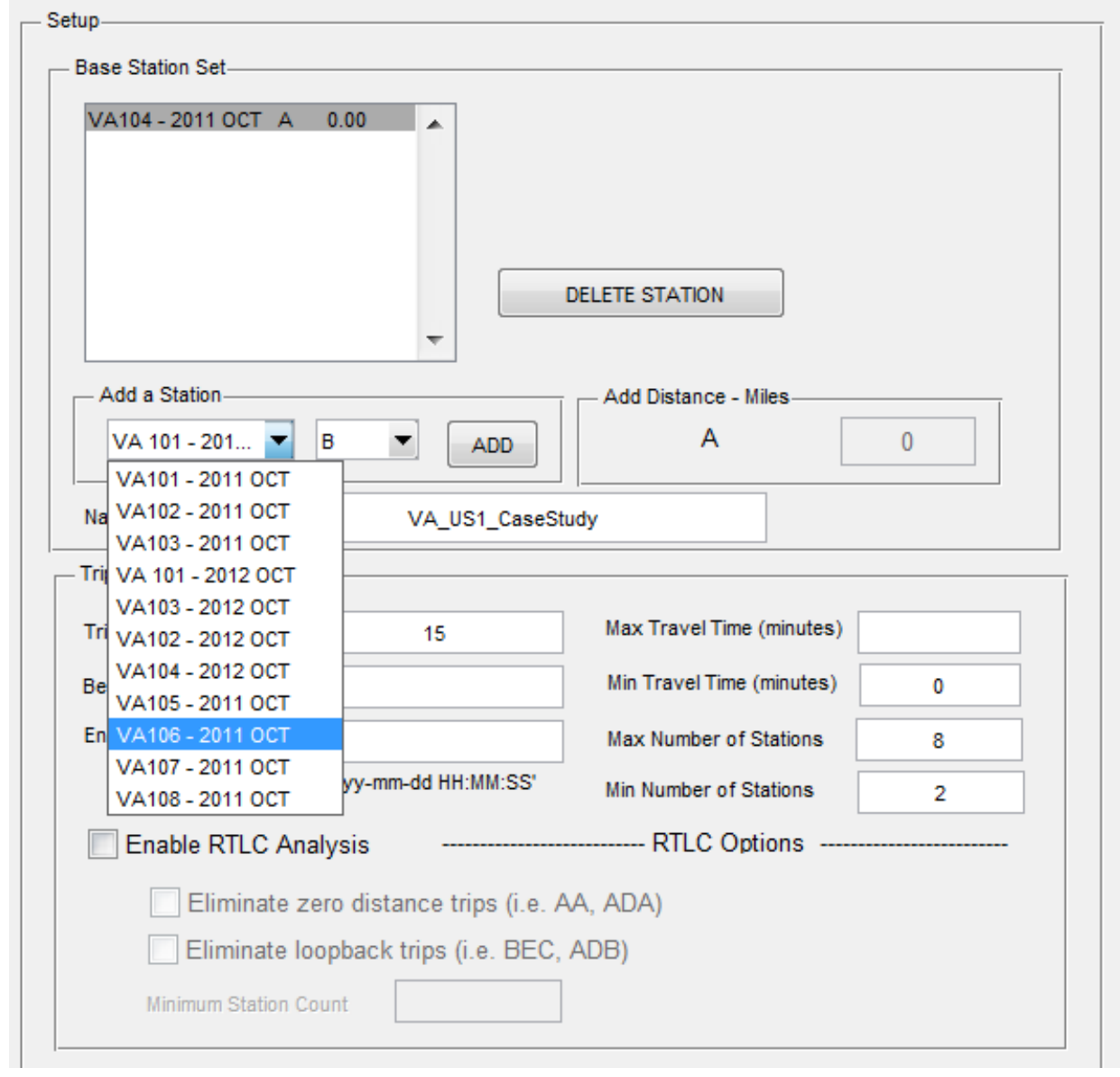

Figure 6 Input of stations and sequence labels

After the stations and sequence labels are entered, a trip analysis needs to be run. The options in the trip analysis parameters are shown in Figure 7. Following is a brief review of these options. A more detailed explanation of the parameters is included in the BluSTATs manual which describes the Trip Analysis base capability.

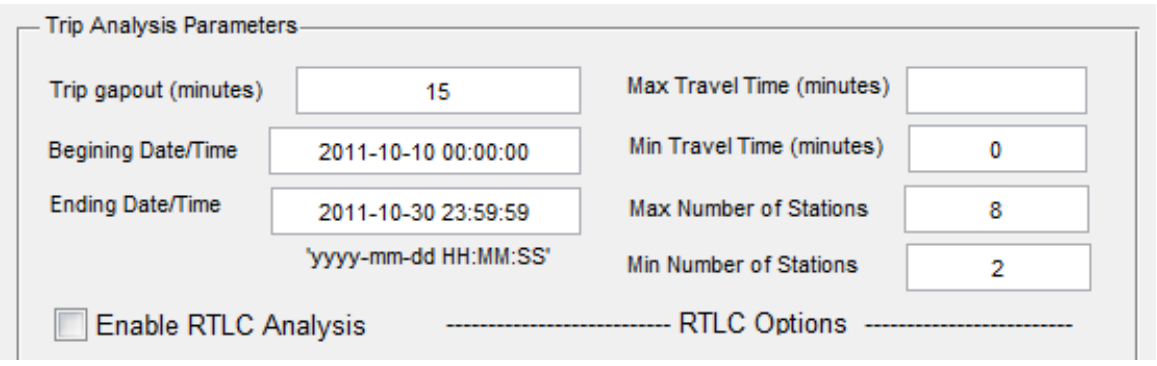

Figure 7 Trip Analysis Parameters 
Trip gapout (minutes): This is a mandatory parameter. This parameter determines the amount of time, measured in minutes, after which a new trip is registered. The default in BluSTATs for generic trip analysis is 15 minutes. Depending on the spacing of the stations and the expected travel time of vehicles through the corridor, this parameter can be adjusted. For example, if sensors are spaced only 0.5 miles apart, then anticipated travel time is never any more than two to three minutes. In such a case, the trip gap-out could be set at five to ten minutes. If the sensors are set 5-10 miles apart, and there is significant congestion such that the maximum anticipated travel time is 20 to 25 minutes, an appropriate Gap Out may be 30 to 45 minutes. Within the software, if a sensor detects a vehicle at one station and does not see that vehicle again at any station for the amount of time set as the gap-out period, the trip chain is terminated.

Beginning Date/Time and Ending Date/Time: These are optional parameters. A beginning or ending date-time may be entered to limit the time expanse of the trip analysis. In this example a datetime limit from Oct 10 through Oct 302011 is entered.

Max Travel Time: This is an optional parameter. If entered, it will discard any trips that exceed the designated travel time.

Min Travel Time: This is an optional parameter. If entered, it will discard any trip that is less than the designated minimum travel time.

Max / Min number of stations: These are optional parameters. If entered, the software will filter out any trip chains that exceed the maximum or are less than the minimum number of stations in a single trip. For RLTC purposes, a minimum of 2 is recommended. This will direct the software to discard any trip chains of only one station.

Once the Trip Analysis is initialized with stations and processing parameters, it should appear as shown in Figure 5. Once entered, the trip analysis can be run.

The trip analysis results in two output listings. The first is labeled the trip chain summary. It contains the listing of unique trip chains found in the data set, ordered by the frequency with which each trip chain appears. An example from this case study is shown in Figure 8. Note that the two most frequent trips are BA and AB with 18449 trip count and 12569 trip counts respectively. Next is followed by FG and GF. After the trip chain and count, the minimum, mean, and maximum trip times are provided. This table is augmented with the trip distance, calculated from the first to the last station in the trip chain. 


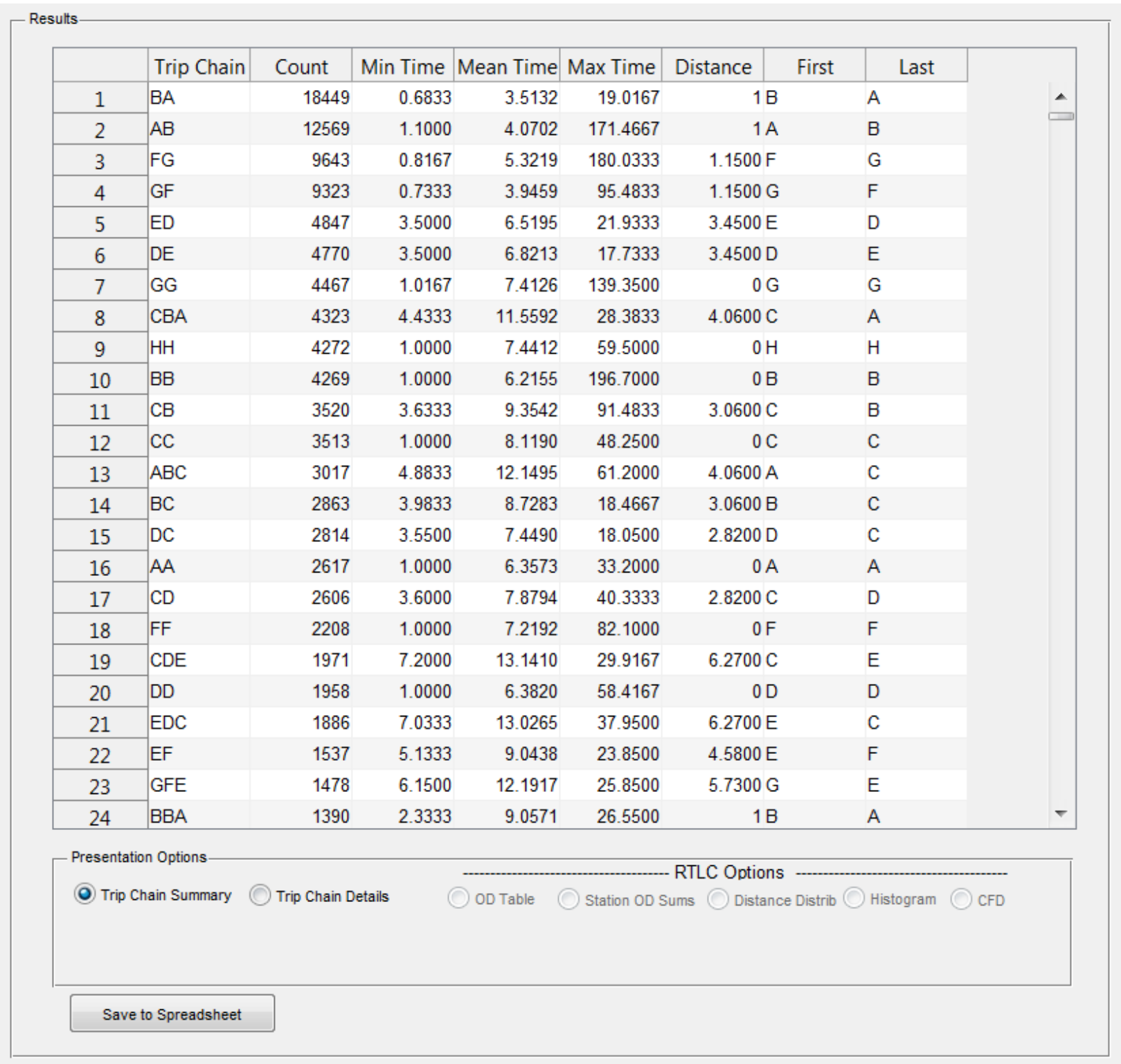

Figure 8 Trip chain summary output

Note the Trip Chain Summary can contain some trips that are unusual. One example of an unusual trip is seen in line 7, GG. This indicates that a vehicle was detected at station $\mathrm{G}$ and then was later detected at station $\mathrm{G}$ within 15 minutes, as determined by the gap-out parameter. Note that the trip travel time ranged from 1 minute to 139 minutes, with an average of 6 minutes. Note that trip chains of only a single detection, such as ' $G$ ' are already filtered. A single detection trip chain, ' $G$ ', could indicate a car that traversed across the corridor, and was detected only once as it crossed. A trip chain of 'GG' may have been a car that traversed across the corridor, stopped briefly perhaps for coffee or at an ATM, and then returned again traversing across the corridor at the same sensor location. This is only one example. There are a number of situations that could cause multiple detections at a single station. However, for RTLC analysis, trip chains such as 'GG' are discarded from the analysis. 
Another example of an unusual trip chain is seen in line 24 with a 'BBA' trip chain. This indicates that the vehicle was originally detected at station $\mathrm{B}$, at some later point in time, but within the 15 minute gap out period, it was detected at station B again, before it was finally detected at station A. As with the previous example, some type of circulation within the corridor could explain this phenomenon. However, for the RTLC analysis, the 'BBA' is treated the same as a 'BA' trip chain. [Note: Though not common, trip chains that appear to skip a station, such as 'ABDE', will appear in the trip chain summary. These occur primarily because the probability for detecting a Bluetooth device is not $100 \%$, but typically greater than $90 \%$. As a result, sometimes trip chains are registered that appear to have skipped a station. For RTLC purposes, any missing intermediary stations are inferred. For example, a trip chain of 'ABDE' is treated as if it were 'ABCDE'.]

The Trip Chain Details page provides an exhaustive listing of all trip chains assessed, as shown in Figure 9. The output is ordered by the identifier. The begin date/time and trip time in minutes is provided.

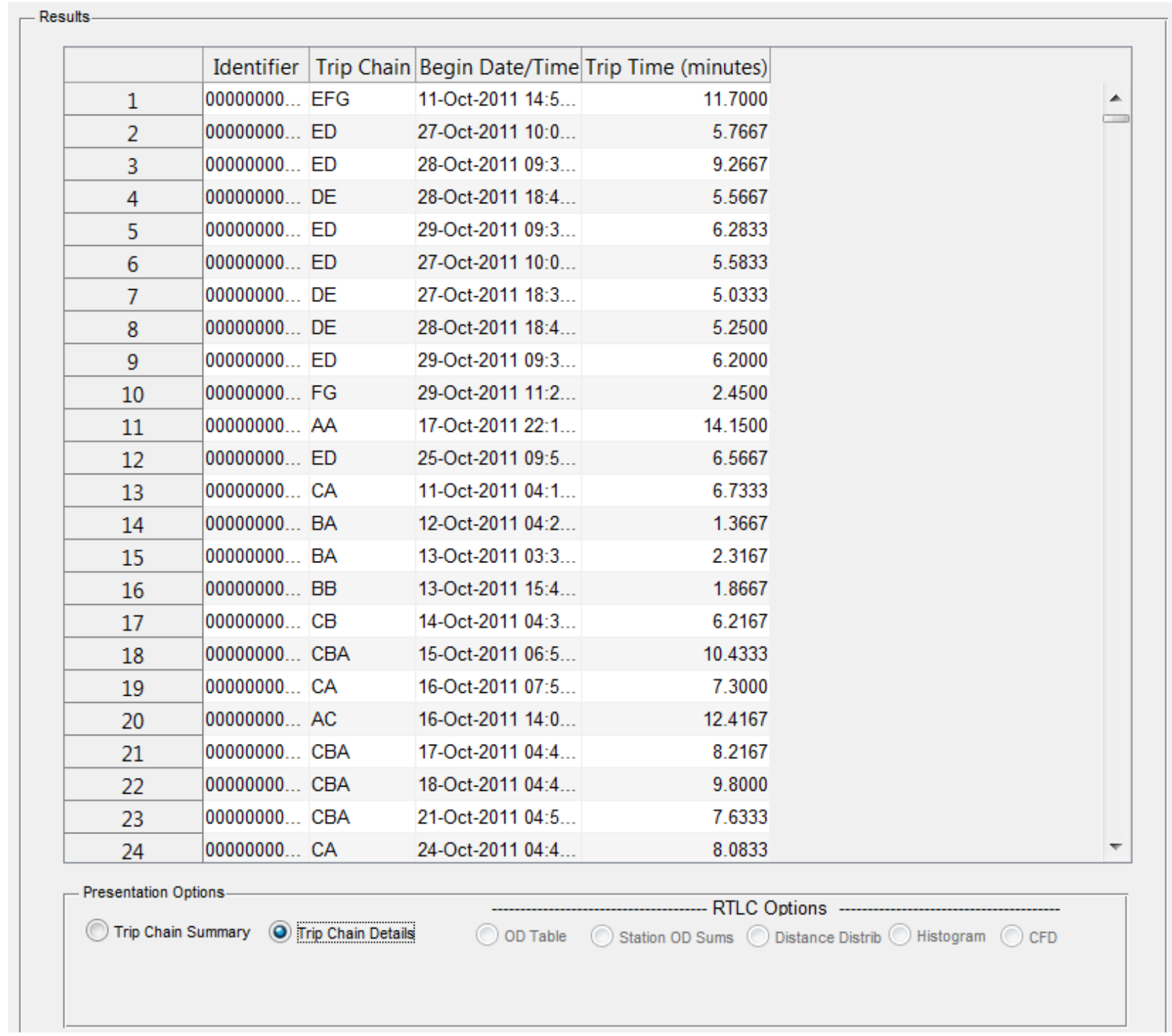

Figure 9 Trip Chain Details example output 
Both the Trip Chain Summary and the Trip Chain Details can be exported to a spreadsheet.

The processing algorithm for assessing trip chains is illustrated in Table 2. Here, detection records are combined into a single table sorted by detected vehicle ID and then by time of detection (first two columns) with the station ID in the third column using the shorthand notation of A,B,C,D...etc.

The next two columns are flags determined by the analysis of the first three data columns. If the vehicle ID changes from the previous record, the 'New Vehicles ID' flag is set. If there is a gap out, that is the time between successive detections exceeds the trip gap-out time parameter, the 'Time Gapout' flag is set. Note that if the 'New Vehicle ID' flag is set, the 'Time Gapout' flag is not assessed (see $10^{\text {th }}$ row as an example).

The remaining columns are logical operators on the flags to determine the beginning and ending of trip chains and associated trip times. If the 'New Vehicle ID' flag is set OR the 'Time Gapout' flag is set for a particular vehicle detection, it indicates the beginning of a new trip chain at that row. Similarly, if the 'New Vehicle ID' flag OR the 'Time Gapout' flag is set for a particular detection, it indicates the ending of a trip chain at the previous row. With knowledge of the beginning and ending location of the trip chain, the appropriate label and trip times can be assigned as shown in Table 2.

Table 2 Trip Chain Processing Logic

Station Data
\begin{tabular}{|c|c|c|c|c|c|c|c|c|}
\hline $\begin{array}{c}\text { Detected } \\
\text { Vehicle ID }\end{array}$ & Detection datetime begin & Station ID & $\begin{array}{c}\text { New } \\
\text { Vehicle ID }\end{array}$ & $\begin{array}{c}\text { Time } \\
\text { Gapout }\end{array}$ & $\begin{array}{c}\text { Begin } \\
\text { Chain }\end{array}$ & $\begin{array}{c}\text { End } \\
\text { Chain }\end{array}$ & $\begin{array}{c}\text { Trip Chain } \\
\text { Trip Time } \\
\text { (mm:ss) }\end{array}$ \\
\hline 0013B2597D4C & $10 / 12 / 113: 12: 34$ PM & A & $\mathrm{X}$ & & $\checkmark$ & & ABCD & $46: 27$ \\
\hline 0013B2597D4C & $10 / 12 / 113: 15: 24$ PM & B & & & & & & \\
\hline 0013B2597D4C & $10 / 12 / 113: 21: 51$ PM & C & & & & & & \\
\hline 0013B2597D4C & $10 / 12 / 113: 59: 01$ PM & D & & & & $\checkmark$ & & \\
\hline 0013B2597D4C & $10 / 14 / 114: 12: 34$ PM & F & & $X$ & $\checkmark$ & & FE & $11: 00$ \\
\hline 0013B2597D4C & $10 / 14 / 114: 23: 34$ PM & E & & & & $\checkmark$ & & \\
\hline OFF122B78C23 & $10 / 12 / 116: 27: 21$ AM & B & $X$ & & $\checkmark$ & & BCD & $07: 41$ \\
\hline OFF122B78C23 & $10 / 12 / 116: 32: 34$ AM & C & & & & & & \\
\hline OFF122B78C23 & $10 / 12 / 116: 35: 02$ AM & D & & & & $\checkmark$ & & \\
\hline 4GF76B007632 & $10 / 21 / 1110: 01: 23$ PM & E & X & & $\checkmark$ & & EDC & $20: 32$ \\
\hline 4GF76B007632 & $10 / 21 / 1110: 12: 43$ PM & D & & & & & & \\
\hline 4GF76B007632 & $10 / 21 / 1110: 21: 55$ PM & C & & & & $\checkmark$ & & \\
\hline
\end{tabular}




\subsection{Software Implementation - RTLC Filtering}

The Roadway Trip Length Characteristic (RTLC) operates on the information in the trip chains but requires additional information. Note that the information presented in the trip chain summary output in Figure 8 contains distance information. Distance between stations is required for RTLC analysis. Distance is not normally required for trip chain analysis, but it is required for RTLC. The distances entered are the distance along the corridor from one station to the next. These can be approximated with straight line distances using geospatial coordinates of the stations as the end points though the software also needs the ability for the user to overwrite straight line distance approximations, as shown in the interface depicted in Figure 5.

Once distance information is initialized, the trip chain summary can be augmented with columns showing the trip distance as shown in Figure 8. Trip distance is calculated from the beginning station to the end station for each trip chain. For example, the trip chain ABCD would have a trip length that is the sum of the individual segments $\mathrm{AB}, \mathrm{BC}$, and $\mathrm{CD}$.

Additional filtering options are introduced for the RTLC as shown in Figure 10.

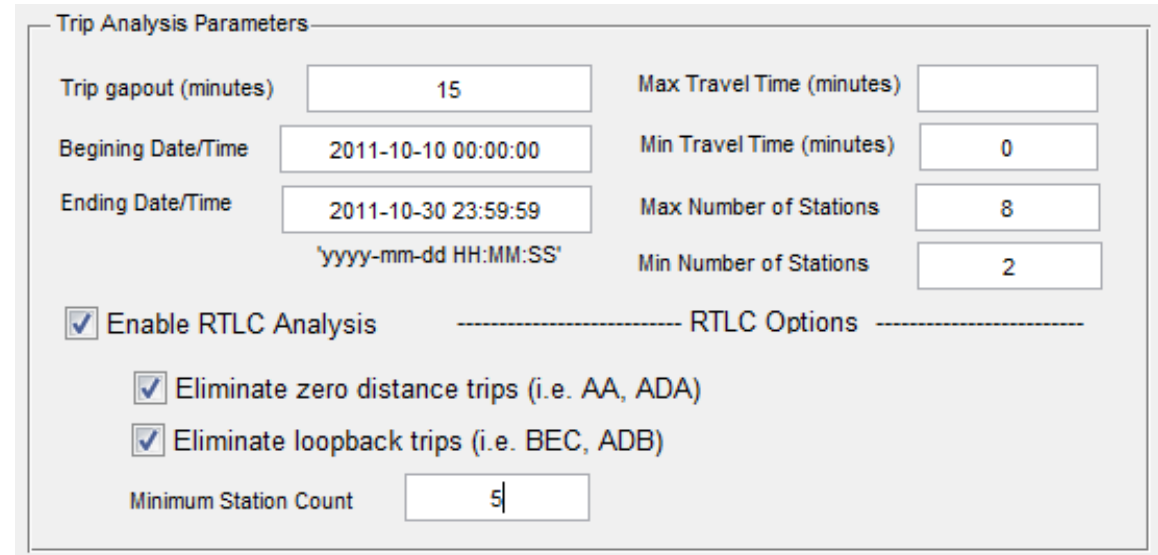

Figure 10 Additional filtering options for the RTLC analysis

These filtering options include:

Eliminate zero distance trips: This option filters out any trip that begins and ends at the same station. Examples of trip chains of this type include AA or BBB for trip chains with only a single unique station. Trip chains with multiple stations, which still begin and end at the same station are also filtered such as ABA or EEFGHGHE.

Eliminate loop back trips: This option filters out any trip chains that represent some type of turnaround in the corridor (or loop back). An example of such a trip chain would be ABCB, DCBABC, or ABA. Note that ABA would be filtered by either the 'Eliminate zero distance trips' filter or the 'Eliminate loop back trips' filter.

Minimum trip count: This parameter trims the trip table to only those trip chains observed for a minimum number of occurrences. For example, if the trip chain ABDEF is observed four times, 
and the minimum station count is set to five, it will be omitted from the Trip Chain Summary table, as well as any subsequent processing to obtain the RTLC.

The RTLC can be calculated without any of the three additional filters discussed above. However, all three filters are recommended to reduce the noise and eliminate data not reflective of how vehicles traverse a corridor. Zero distance trips are typically noise in the system, such as vehicles that cut across the corridor, and then return, as well as pedestrians and cyclists that circulate near a particular station. Trips that loop back in the corridor may arise from a number of phenomena, not only U turns, but also vehicles that exit the roadway and loop back around on parallel streets. The minimum trip count reduces the trip chains summary table to only those observed on a regular, repeated basis. The minimum trip count is typically set from 5 to 50 depending on the size of the data set and the level of detail desired in the reports. All trips (Minimum Trip Count set to 1) may also be chosen, but this will create exceptionally long trip chain summary tables.

An example of a typical initialization using the US1 case study from Northern Virginia is shown in Figure 11 prior to processing.

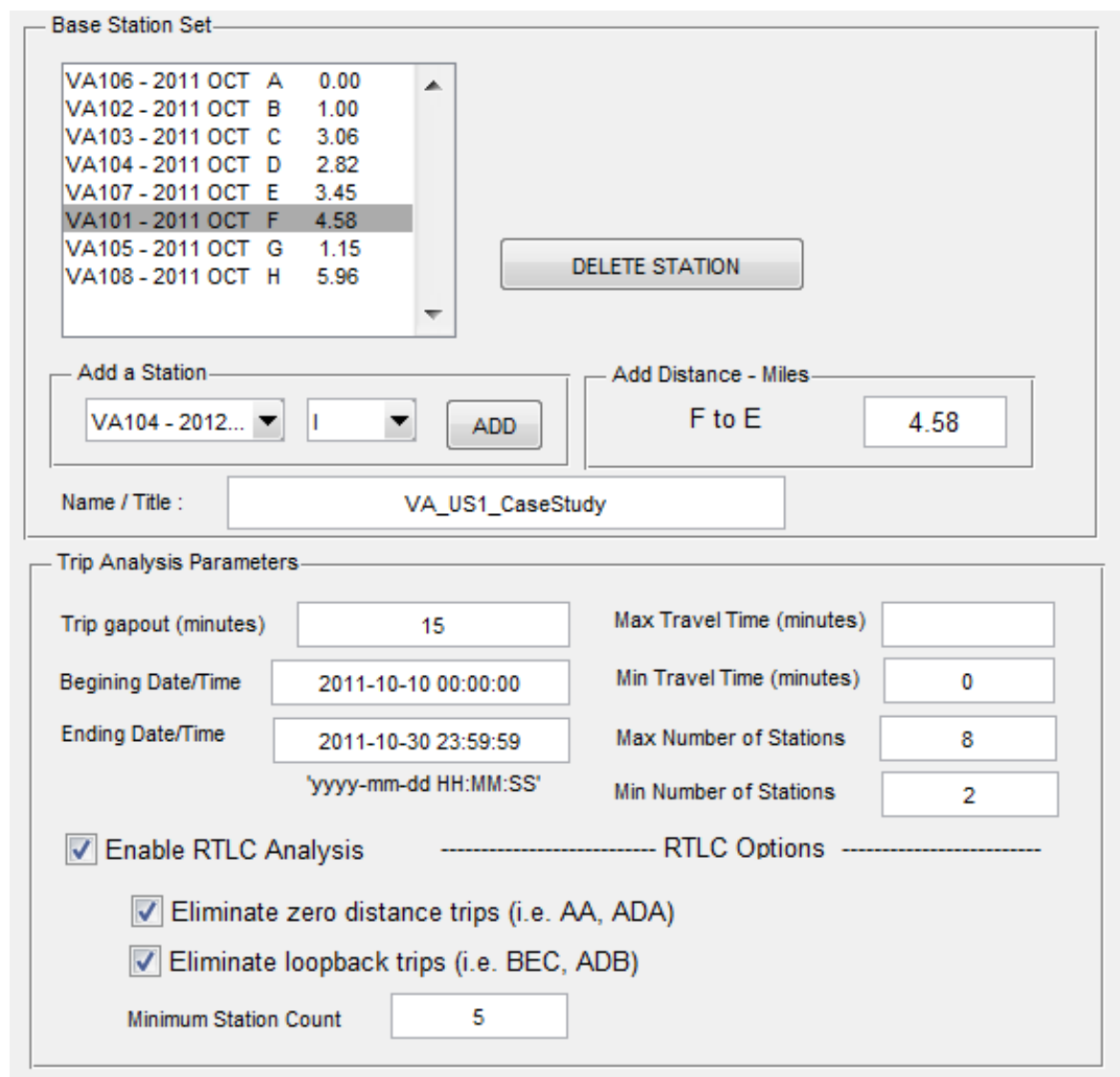

Figure 11 Trip Analysis and RTLC options for an RTLC analysis for US1 case study

The trip chain summary for this analysis is shown in Figure 12. Contrasted with the trip chain summery in Figure 8, prior to the introduction of additional RTLC filters, the summary in Figure 12 has no zero length trip chains in the abbreviated listing shown. If the whole trip chain summary listing were 
viewable, the user would note that loop-back chains were removed as well as any trip chains with less than five observances. In total, prior to the additional RTLC filters, the trip chain summary contained 1345 entries. After the RTLC additional filters, the total number of unique trip chains was reduced to 324.

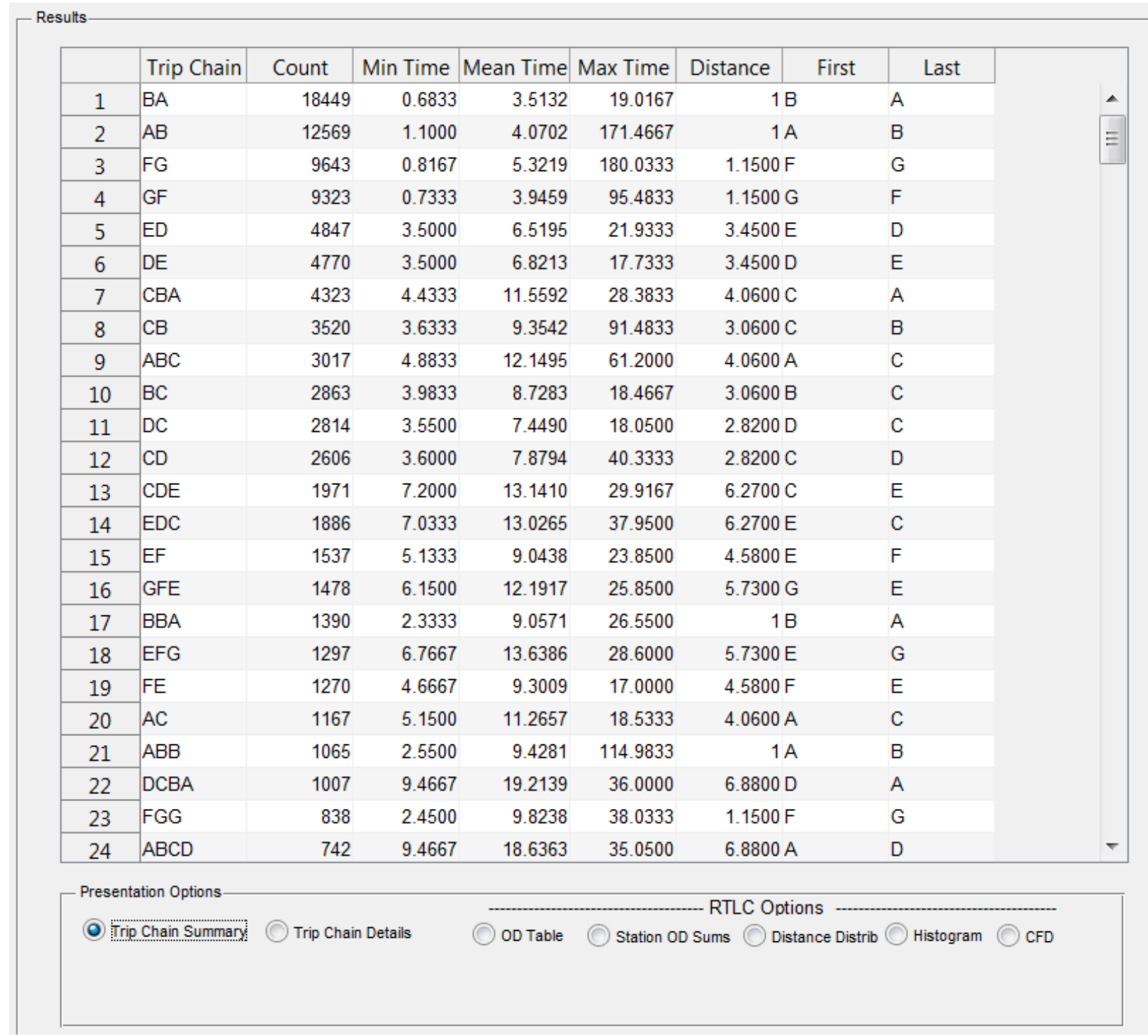

Figure 12 Trip chain summary table for US-1 with RTLC filter options applied

Figure 13 provides an Origin-Destination (OD) table for the trips along the US1 corridor. Note the main diagonal of all zeros, consistent with the zero distance trip filter application. Such OD tables are typically symmetric, with roughly the same number of trips between any two points in opposite direction when viewed over a multi-day period. If the RTLC analysis is confined to either a morning or afternoon peak period, the symmetry in the OD table would likely be broken due to the tidal flows associated with journey to or from work patterns. 


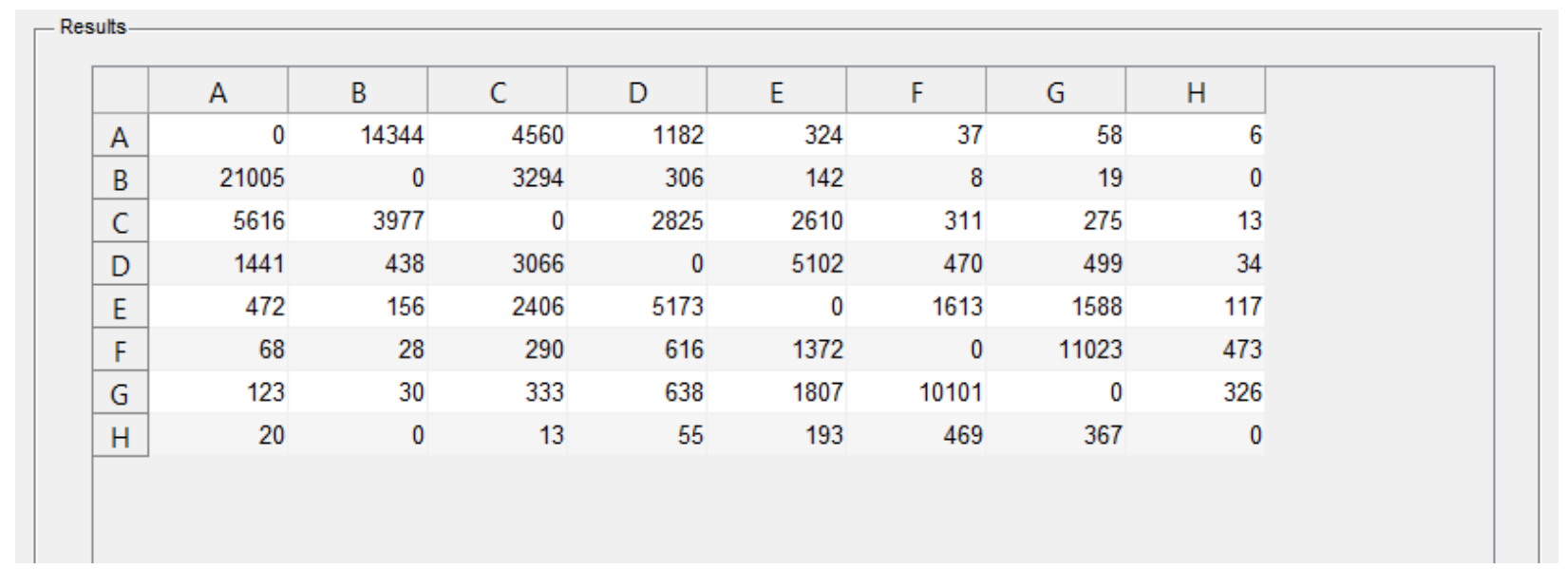

Figure 13 Origin-Destination table of trips for the US-1 corridor

Figure 14 provide an origin-destination summary by station. This table lists the frequency in which any station is the origin (first column), destination (second column), or an interior (through) node in the trip chain (third column).

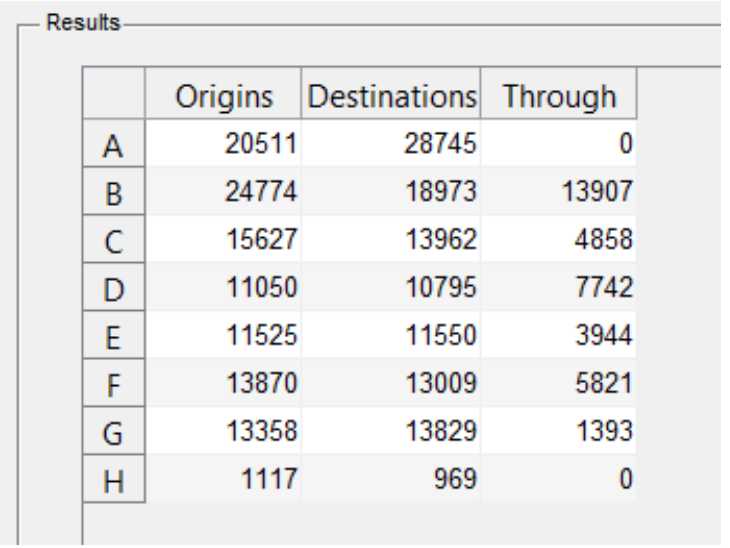

Figure 14 Origin-Destination summary by station

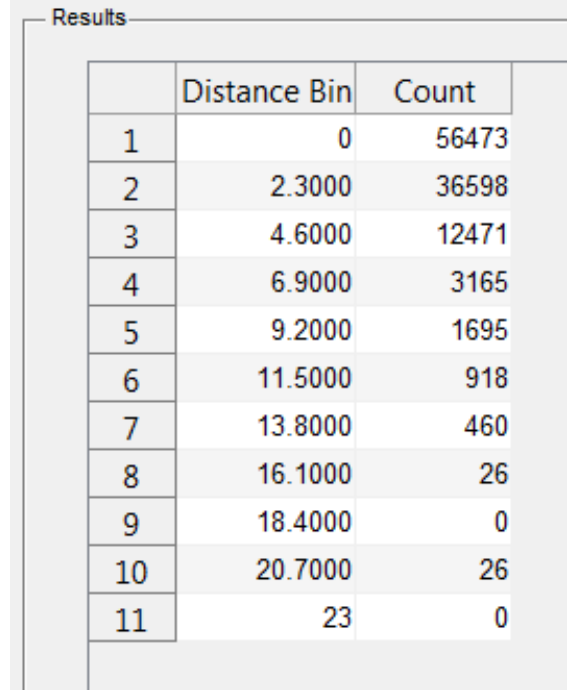

Figure 15 Distance distribution table

Figure 15 provides a table for the distance distribution in the corridor. For each trip in the OD table, the frequency of the trip combined with the distance between the origin and destination determines the appropriate distance bin. For example, any trips from station A to station C or station C to station A are a distance of 4.06 miles each, based on information in Table 1. The number of trips from A to $\mathrm{C}$ totals 4560 and from $C$ to A totals 5616. Both of these are tallied in bin two representing trips of 2.3 to 4.6 miles. All trips in this range (which include A to C and C to A as well as many others), total 36598 trips. These are standard histogram tables. In this case study, ten distance bins were used.

Figure 16 and Figure 17 are visualizations of the data from the distance distribution table shown in Figure 15 . Figure 16 provides a normalized histogram view. Rather than absolute number of observations on 
the y axis, the percentage of all trips in each bin is provided. For US-1, approximately half of all trips are in the first bin, which is less than 2.3 miles in length. This reflects that US-1 is used primarily to access the various stores and services along the route.

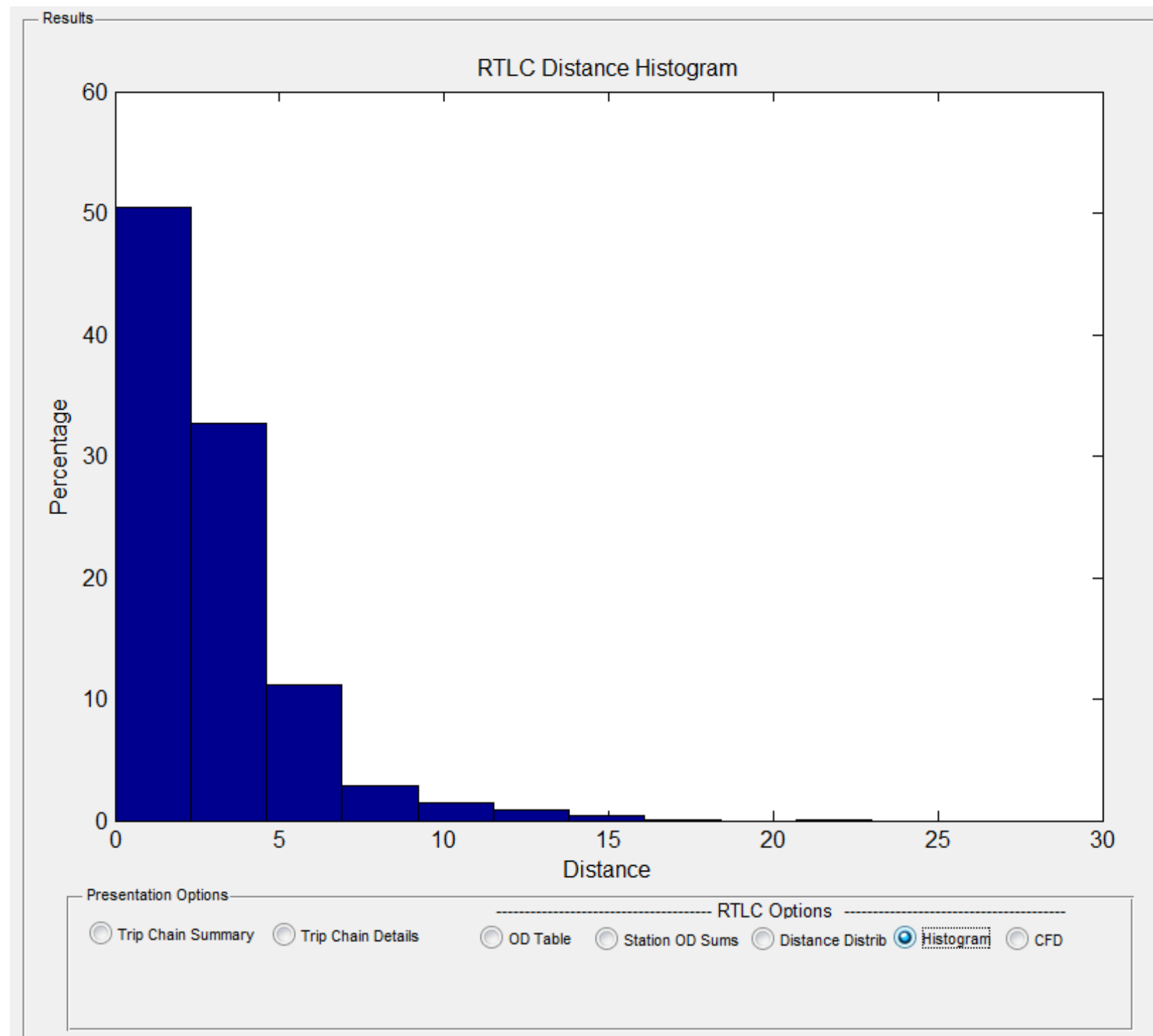

Figure 16 Histogram view of the RTLC trip distance distribution for US-1

Figure 17 provides the same information as Figure 16, but in a compact Cumulative Frequency Diagram (CFD) view. This view allows for direct comparison of the RTLC from different corridors, before and after analysis, or for different time periods. 


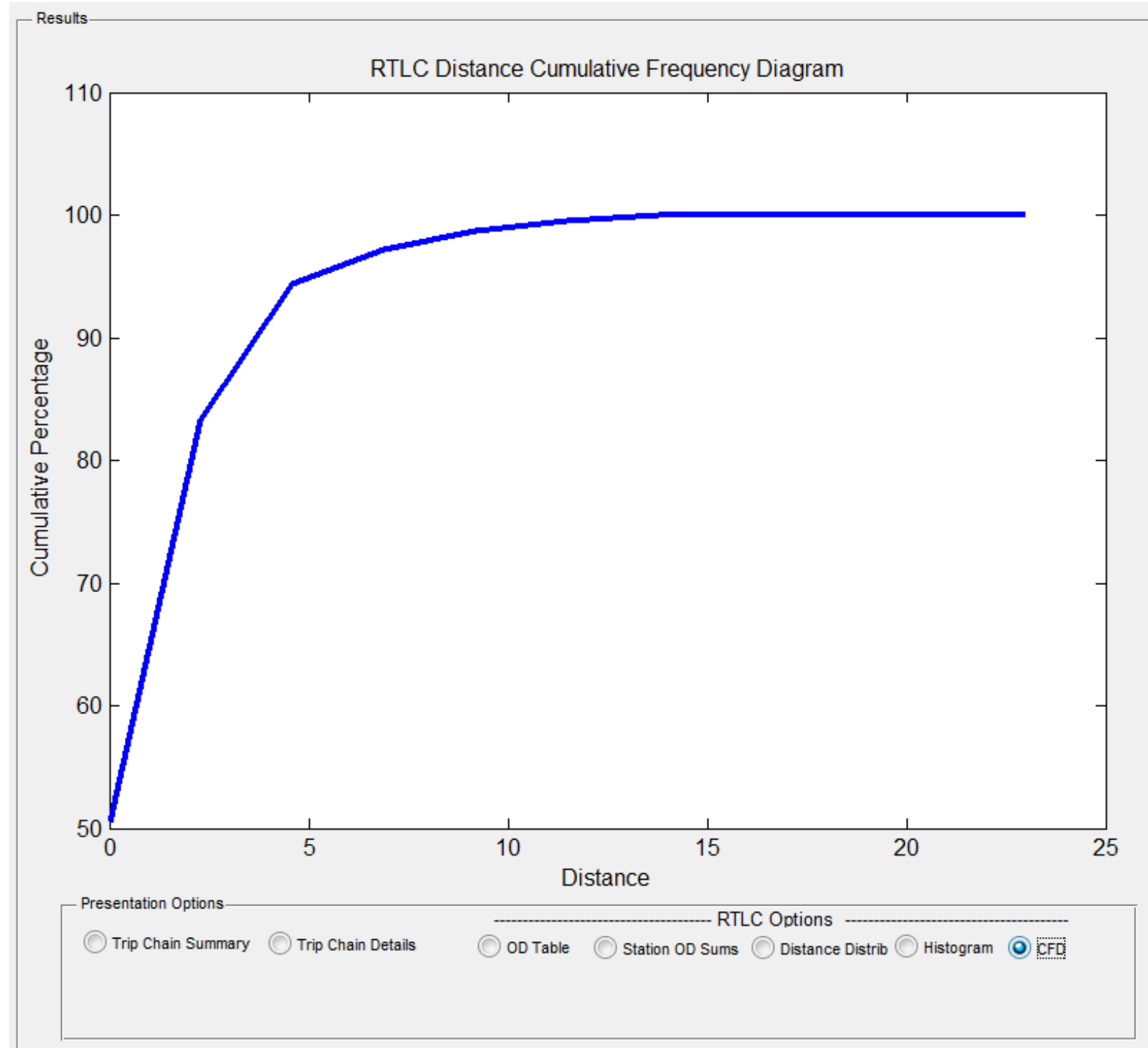

Figure 17 Cumulative Frequency Diagram (CFD) view of the RTLC 


\subsection{Summary}

The Roadway Trip Length Characteristic (RTLC) has been programmed into the BluSTATs analysis program distributed by Traffax Inc. It operationalizes the RTLC statistics provided in previous research for use on data collected in the field. This report provides a detailed explanation of the processing sequence and algorithms needed for implementation.

The RTLC process relies on a trip analysis in which the vehicles are observed as they progress through a series of stations, resulting in what are referred to as trip chains. Trip chains are denoted in a short-hand format. Each station along a corridor is assigned a letter in the order of progression along the corridor. The first station is assigned ' $A$ ', the second ' $B$ ', the third ' $C$ ' and so on. The short hand notation provides the path taken on the corridor. The trip chain 'BCD' indicates the vehicle was first observed at station $\mathrm{B}$, then $\mathrm{C}$, and lastly $\mathrm{D}$. The analysis of trip chains results in a tabulated list of unique trips observed, and the total number of observances of that trip. An example of the trip chain summary table is shown in Figure 12.

The RTLC uses the information in the trip chain summary combined with the calculated distance of each trip chain based on the beginning and ending station. Note that additional filters are typically applied during an RTLC analysis, over and above the filters used in the trip analysis. These filters eliminate zero length trips, loop-back trips, and omit trip chains observed below a minimum threshold. These filters eliminate noise and outliers in the data and reduce the overall size of the resulting trip chain summary table, making reporting more manageable. The data in the trip chain summary table is then transformed into an OD summary table as shown in Figure 13. The data in Figure 13, combined with the trip distances, provides for the construction of trip length distribution within the corridor (see Figure 15), from which a visualization in either histogram form (Figure 16) or CFD form (Figure 17) can characterize how the corridor is used by providing a direct measure of its access versus throughput properties.

Note that the implementation of the RTLC operates on station detection records, not on segment travel time records available in the CWS5200 segment data standard. This implementation exposed the need to define a station detection data standard, comparable to CWS5200, but applicable to individual detections at stations.

This report is intended as a reference document for the implementation of the RTLC for procurement of equipment and analytics related to re-identification data. Please contact Traffax Inc. for any clarifications or extensions of the RTLC reference implementation and case study herein. The RTLC software, as demonstrated, is available in BluSTATs version 2.25 and above. 


\subsection{Bibliography}

[1] D. S. T. F. Stanley E. Young, "Arterial Trip Length Characteristics," SBIR Phase 3 Federal Highway Administration Project "Sensor Fusion and MOE Development for Off-Line Traffic Analysis of Real Time Data, Septemer 2, 2016. 


\section{Report Sponsor}

The "Small Business Innovation Development Act of 1982" (Pub. L. No. 97-219), along with reauthorizing legislation (Pub. L. No. 99-443 and Pub. L. No. 102-564, the "Small Business Research and Development Enhancement Act of 1992"), seeks to encourage the initiative of the private sector and to use small business effectively to meet federal research and development objectives. To comply with statutory obligations of the Act, the U.S. Department of Transportation established the Small Business Innovation Research (SBIR) Program, which conforms to the guidelines and regulations provided by the Small Business Administration. Annually, small businesses are solicited to submit innovative research proposals that address the high-priority requirements of the U.S. Department of Transportation and that have potential for commercialization.

This report was developed through a partnership between Traffax, Inc., and Purdue University with funding from a Phase III SBIR contract (DTFH6114C00035) with the Federal Highway Administration. The project, entitled "Sensor Fusion and MOE Development for Off-Line Traffic Analysis of Real Time Data," created and refined methods and tools for the characterization of performance along arterial corridors.

\section{Publication}

This report is part of a series of reports published in collaboration with USDOT, Traffax, Inc., and Purdue University. The full report series is available for download at http://docs.lib.purdue .edu/apmtp/.

\section{Open Access and Collaboration with Purdue University}

The Indiana legislature established the Joint Highway Research Project in 1937. In 1997, this collaborative venture between the Indiana Department of Transportation and Purdue University was renamed as the Joint Transportation Research Program (JTRP) to reflect state and national efforts to integrate the management and operation of various transportation modes. Since 1937, the JTRP program has published over 1,600 technical reports. In 2010, the JTRP partnered with the Purdue University Libraries to incorporate these technical reports in the University's open access digital repository and to develop production processes for rapidly disseminating new research reports via this repository. Affiliated publications have also recently been added to the collection. As of 2017, the JTRP collection had over 1.5 million downloads, with some particularly popular reports having over 20,000 downloads. 